\title{
Dietary flavonoid fisetin targets caspase-3-deficient human breast cancer MCF-7 cells by induction of caspase-7- associated apoptosis and inhibition of autophagy
}

\author{
PEI-MING YANG ${ }^{1}$, HO-HSING TSENG ${ }^{2}$, CHIH-WEN PENG ${ }^{2}$, WEN-SHU CHEN ${ }^{2}$ and SHU-JUN CHIU ${ }^{2}$ \\ ${ }^{1}$ Department of Pharmacology, College of Medicine, National Taiwan University, Taipei; \\ ${ }^{2}$ Department of Life Science, Tzu Chi University, Hualien, Taiwan, R.O.C.
}

Received July 15, 2011; Accepted August 30, 2011

DOI: $10.3892 /$ ijo.2011.1203

\begin{abstract}
The outcome of producing apoptotic defects in cancer cells is the primary obstacle that limits the therapeutic efficacy of anticancer agents, and hence the development of novel agents targeting novel non-canonical cell death pathways has become an imperative mission for clinical research. Fisetin (3,3',4',7-tetrahydroxyflavone) is a naturally occurring flavonoid commonly found in fruits and vegetables. In this study, we investigated the potential anticancer effects of fisetin on breast cancer cells. The result showed fisetin induced higher cytotoxicity in human breast cancer MCF-7 than in MDA-MB-231 cells otherwise it did not exert any detectable cytotoxicity in non-tumorigenic MCF-10A cells. We found fisetin can trigger a novel form of atypical apoptosis in caspase-3-deficient MCF-7 cells, which was characterized by several apoptotic features, including plasma membrane rupture, mitochondrial depolarization, activation of caspase-7, -8 and -9, and PARP cleavage; however, neither DNA fragmentation and phosphotidylserine (PS) externalization was observed. Although p53 was also activated by fisetin, the fisetin-induced apoptosis was not rescued by the p53 inhibitor pifithrin- $\alpha$. In contrast, the fisetin-induced apoptosis was abrogated by pan-caspase inhibitor z-VAD-fmk. Furthermore, inhibition of autophagy by fisetin was shown as additional route to prompt anticancer activity in MCF-7 cells. These data allow us to propose that fisetin appears as a new potential anticancer agent which can be applied to develop a clinical protocol of human breast cancers.
\end{abstract}

\section{Introduction}

Apoptosis and necrosis are known as the two major routes leading to cell death $(1,2)$. Apoptosis is generally characterized

Correspondence to: Dr Shu-Jun Chiu, Department of Life Science, Tzu Chi University, 701, Section 3, Chung-Yang Road, Hualien 970, Taiwan, R.O.C.

E-mail: chiusj@mail.tcu.edu.tw

Key words: flavonoid, fisetin, human breast cancer, apoptosis, autophagy by distinguishable morphological features, such as cytoplasmic shrinkage, chromatin condensation, phosphatidylserine (PS) externalization, DNA fragmentation, and formation of apoptotic bodies without affecting plasma membrane integrity (3), whereas necrosis is defined on the basis of other morphological criteria, including pyknotic nuclei, cytoplasmic vacuolation, breakdown of the plasma membrane, and induction of an inflammatory response as a result of the release of cellular contents (2). Although necrosis has long been considered as an accidental process, this type of cell death can occur by a genetically controlled (programmed) process (4).

Autophagy is a physiological process involved in the turnover of proteins or intracellular organelles through lysosomal machinery (5). The process of autophagy starts by sequestering cytosolic proteins or organelles into autophagosomes which is then fused with lysosomes to form autolysosomes for subsequent degradation of sequestered contents by lysosomal hydrolases (6). The two-faced roles of autophagy were characterized with its anti-tumor effects through promoting autophagic cell death (type II programmed cell death) and survival-promoting function in starved cells (6). Thus, inhibition of cytoprotective effects induced by autophagy and promotion of autophagic cell death might be employed as new strategies for anticancer therapy.

Breast cancer is the most common malignancy found in American and Northwestern European women $(7,8)$. Breast epithelial cells require a concomitant balance between cell proliferation and apoptosis under a physiological environment. Apoptosis occurs in healthy breast cells at fluctuating rates during the estrus cycle in response to changes of hormone levels $(9,10)$. However, defects in the apoptotic regulatory pathway may result in the accumulation of genetically altered cells, leading to tumorigenesis (10). Induction of apoptosis in tumor cells is initially thought as an effective protocol for anticancer therapy; however, cells can subsequently survive and gain resistance to these treatments through acquisition of apoptotic resistance (11). Consequently, it becomes an urgent need to develop an effective protocol of cancer therapy to overcome apoptotic resistance.

The natural flavonoid fisetin (3,7,3',4'-tetrahydroxyflavone) (Fig. 1A), which is found widely in fruits and vegetables, has been previously shown to exhibit anticancer activities in various types of tumor cells, with the capability of inducing cell cycle 
arrest and apoptosis (12-17). Nevertheless, the crucial anticancer effects of fisetin in human breast cancer cells remain unclear. In this study, the cytotoxic and apoptotic effects induced by fisetin in human breast cancer MCF-7 and MDA-MB-231 cells were extensively investigated. Interestingly, fisetin was found to exhibit a robust anticancer activity in caspase-3-deficient MCF-7 cells. In addition, fisetin-induced apoptosis did not display these typical features of apoptosis such as DNA fragmentation and PS externalization and instead triggered plasma membrane rupture, mitochondrial depolarization, activation of caspase-7, -8 and -9 , and PARP cleavage in MCF-7 cells, which can be intensively blocked by caspase inhibition. Furthermore, inhibition of autophagy by fisetin substantially promoted the occurrence of cell death in MCF-7 cells. Taken together, our results suggested that fisetin can be used as a potential candidate for developing the therapeutic protocol of human breast cancers in the future.

\section{Materials and methods}

Cell culture and reagents. MCF-7 and MDA-MB-231 human breast cancer cell lines were routinely maintained in RPMI1640 medium (Gibco) and Dulbecco's modified Eagle's medium (DMEM; Gibco), and the complete medium was supplemented with 10\% fetal bovine serum. MCF-10A human breast epithelial cells were kindly provided by Dr Ho Yuan-Soon (School of Medical Laboratory Science and Biotechnology, Taipei Medical University, Taiwan), and these cells were maintained in DMEM/ F12 medium (Gibco). Fisetin (3,3',4',7-tetrahydroxyflavone), Necrostatin-1 (Nec-1), 3-(4,5-dimethylthiazol-2-yl)-2,5-diphenyl tetrazolium bromide (MTT), propidium iodide (PI), 5, 5',6, $6^{\prime}$ tetrachloro-1,1',3,3'-tetraethylbenzimidazolcarbocyanine iodide (JC-1), pifithrin- $\alpha$, 3-methyladenine (3-MA) and bafilomycin A1 were purchased from Sigma-Aldrich. z-VAD-fmk was purchased from Bachem. Antibodies specific to LC3B, poly(ADP-ribose) polymerase (PARP), cleaved caspase-7, -8 and -9 , phospho-p53 (Ser15), p53(DO-1), and goat anti-rabbit IgGhorseradish peroxidase were obtained from Cell Signaling Technology. Anti-actin antibody was purchased from Chemicon International.

Cell viability assay. Cell viability was determined by MTT colorimetric assay. Briefly, cells were seeded at a density of 40,000 cells per well in 6-well plates, and each well was aliquoted with $2 \mathrm{ml}$ of culture medium. Cells were pretreated with inhibitors for $2 \mathrm{~h}$, washed with PBS, and re-cultured in culture medium for 5-7 days after treatment with fisetin for $24 \mathrm{~h}$. Subsequently, the medium was replaced by new medium supplemented with $0.5 \mathrm{mg} / \mathrm{ml}$ MTT and incubated for $4 \mathrm{~h}$. The viability of cells was determined by the measurement of formazan production converted from MTT, which can be quantified by the development of a blue-purple color in dimethyl sulfoxide (DMSO). The intensity of formazan staining was measured at $545 \mathrm{~nm}$ using a plate reader (Molecular Dynamics, OPTImax), and the relative percentage of viable cells was calculated by dividing the absorbance resulting from the treated cells (the average of six wells) by that of the control included in each experiment.

Apoptosis, PI uptake assay and mitochondrial membrane depolarization studies. Apoptotic cells were examined using an
Annexin V-FITC/PI staining kit (BioVision) according to the manufacturer's instruction. To measure PI uptake, cells were harvested after treatment with the indicated concentrations of fisetin and incubated in culture medium containing $2 \mu \mathrm{g} / \mathrm{ml}$ of PI. The changes of mitochondrial membrane potential (MMP) were assayed using the voltage-sensitive lipophilic cationic dye JC-1. Cells were analyzed by flow cytometry (BectonDickinson), and the percentages of Annexin-V-positive cells, PI uptake cells and low-MMP cells were calculated using ModFit LT software (Ver. 2.0, Becton-Dickinson).

Luminescence ATP detection assay. MCF-7 cells were seeded in 96 -well microtiter plates at $4 \times 10^{4}$ cells per-well and incubated for $24 \mathrm{~h}$ followed by treatment with fisetin. The amount of ATP was quantified using the ATPlite luminescence assay system according to the manufacturer's instruction (PerkinElmer).

Western blot analysis. The protocol for total cellular protein extraction was as described in our previous study (18). Briefly, equal amounts of total protein (20-60 $\mu \mathrm{g} /$ well) were subjected to electrophoresis using 10-12\% sodium dodecyl sulfate-polyacrylamide gels. The polyvinylidene fluoride (PVDF) membranes to which the proteins were electrophoretically transferred were first blotted with the indicated primary antibody followed by subsequent immune blotting using a corresponding horseradish peroxidase-conjugated secondary antibody. Finally, the protein bands were visualized on X-ray film using an enhanced chemiluminescence detection system (Immobilon ${ }^{\mathrm{TM}}$ Western Chemiluminescent HRP Substrate, WBKLS0500; Millipore).

Statistical analysis. All of the data are represented as the mean \pm standard error of the mean (SEM) from at least three independent experiments. Statistical comparisons were performed by one-way analysis of variance, and further post-hoc testing was conducted using the statistical software GraphPad Prism 4 (GraphPad Software, Inc., San Diego, CA, USA). A p $<0.05$ was considered statistically significant.

\section{Results}

Fisetin exhibits substantial cytotoxicity in caspase-3-deficient MCF-7 human breast cancer cells. Although fisetin has been reported to exhibit some anticancer activities (12-15), its anticancer effects in human breast cancer remains elusive. To verify this possibility, we monitored the potential anticancer activity of fisetin by performing MTT assay using human breast cancer cells (MCF-7 and MDA-MB-231) and non-tumorigenic human breast epithelial cells (MCF-10A) treated with various concentrations of fisetin for $24 \mathrm{~h}$. As shown in Fig. 1B, fisetin efficiently induced cytotoxicity in human breast cancer cells but not in MCF-10A cells, indicating the selective cytotoxicity of fisetin towards cancer cells. Intriguingly, MCF-7 cells were shown to be more sensitive to the cytoxicity induced by fisetin than MDA-MB-231 cells (Fig. 1B). It has been reported that genomic deletion of the caspase-3 gene in MCF-7 cells causes apoptotic defects and chemoresistance $(19,20)$. Accordingly, we found that fisetin increased the population of Annexin V-FITC positive cells in MDA-MB-231 cells but not in MCF-7 cells (Fig. 1C and D). In addition, an increasing amount of PI-positive cells was found in fisetin-treated MCF-7 cells 
A<smiles>O=c1c(O)c(-c2ccc(O)c(O)c2)oc2cc(O)ccc12</smiles>

Fisetin (3,3',4',7-Tetrahydroxyflavone)
B

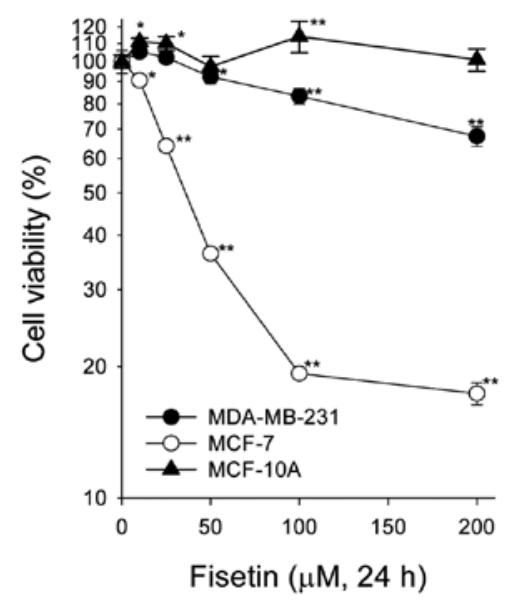

C

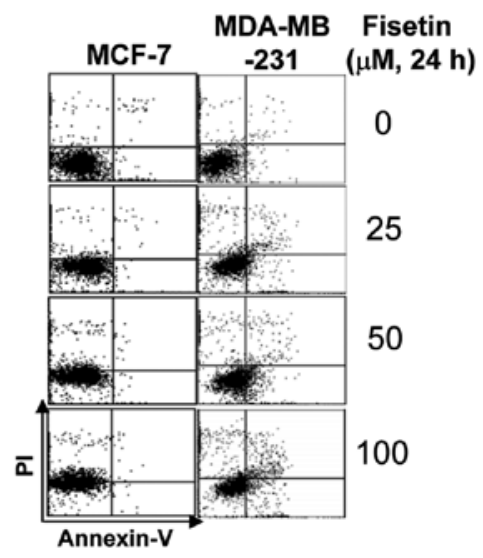

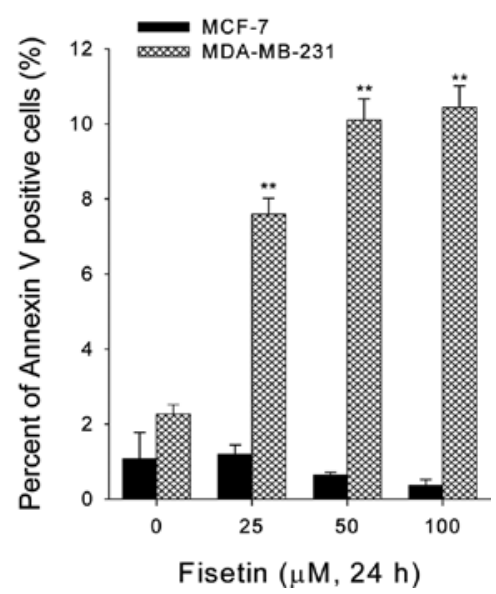

Figure 1. Fisetin induced cytotoxicity and apoptosis in breast cancer cells. (A) The molecular structure of fisetin. (B) MCF-7, MDA-MB-231 and MCF-10A cells were treated with the indicated concentrations of fisetin for $24 \mathrm{~h}$. The treated cells were then subjected to MTT assay. (C) MCF-7 and MDA-MB-231 cells were treated with the indicated concentrations of fisetin for $24 \mathrm{~h}$. Annexin V-FITC/PI double staining was performed. (D) The Annexin V-FITC-positive cells were quantified. The representative ${ }^{*} \mathrm{p}<0.05$ and ${ }^{* *} \mathrm{p}<0.01$ indicate significant differences between fisetin-treated and untreated samples.

(Fig. 1C), suggesting a loss of plasma membrane integrity in fisetin-induced cell death.

Fisetin does not induce necroptosis in MCF-7 cells. The distinctive form of cell death termed necroptosis is characterized by necrotic cell death accompanied by plasma membrane rupture (21). To investigate whether fisetin additionally induced necroptosis in MCF-7 cells, the intracellular ATP levels of fisetin-treated cells were examined. It is known that energy depletion occurs during necrosis but not during apoptosis (22). Our result showed ATP levels were well maintained in the duration of fisetin treatment but strongly declined in response to the necrosis inducer $\mathrm{H}_{2} \mathrm{O}_{2}$ (Fig. 2A), suggesting fisetin-induced death was independent from necrosis. To further verify this hypothesis, the necroptosis inhibitor necrostatin-1 (Nec-1) was utilized to test its ability to rescue the cell viability from cells treated with fisetin (Fig. 2B). Our result demonstrated neither fisetin-induced plasma membrane rupture nor was cytotoxicity altered by Nec-1 (Fig. 2C), indicating fisetin did not induce necroptosis in MCF-7 cells.
Fisetin induces caspase-dependent cell death in MCF-7 cells. Despite the absence of caspase-3 in MCF-7 cells, the presence of other apoptosis executioners such as caspase-7 might act as a functionally compensative candidate for caspase- 3 . To verify whether caspase activation is necessary for fisetin-induced cell death in MCF-7 cells, the effect of pan-caspase inhibitor z-VAD-fmk on the cell death in fisetin-treated MCF-7 cells was then examined. Our result showed that both PI uptake and cytotoxicity induced by fisetin could be rescued by pan-caspase inhibitor z-VAD-fmk (Fig. 3A and B), suggesting fisetin induced a type of caspasedependent cell death in MCF-7 cells. The activation of caspase-7, -8 , and -9 were then monitored based on the measurements of the corresponding proteolytic cleavages shown on the immunoblotted image. Increasing amounts of cleaved caspase-7, -8 , and -9 were observed upon fisetin treatment in a concentration- and time-dependent manner (Fig. 3C and D). Caspase-7 is known to be cleaved by several proteases, including caspase-3, $-6,-8,-9$, and granzyme B (23-25). Moreover, activation of caspase-7 can lead to proteolysis of the many substrates of caspase-3, such as PARP, caspase- 8 and $9(23,26)$. As expected, cleavage of PARP 
A

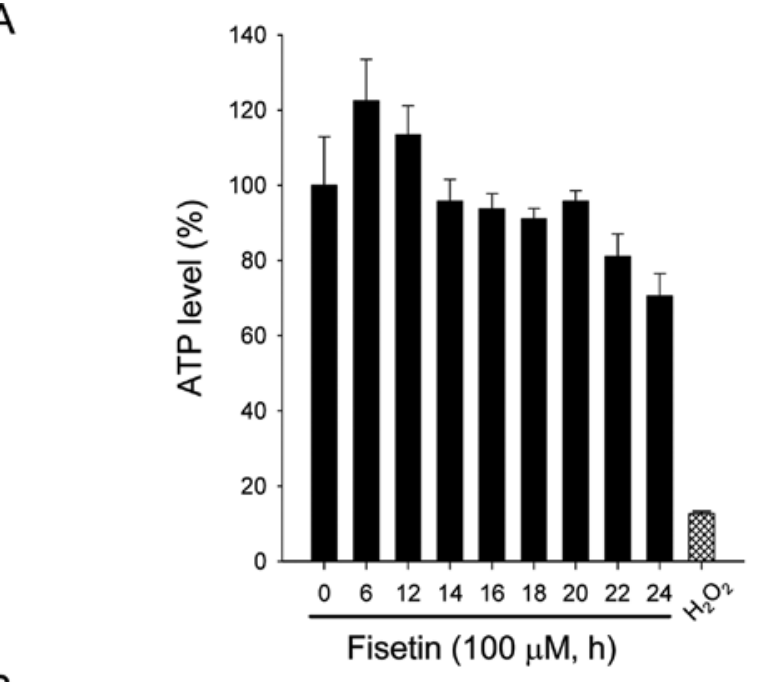

B
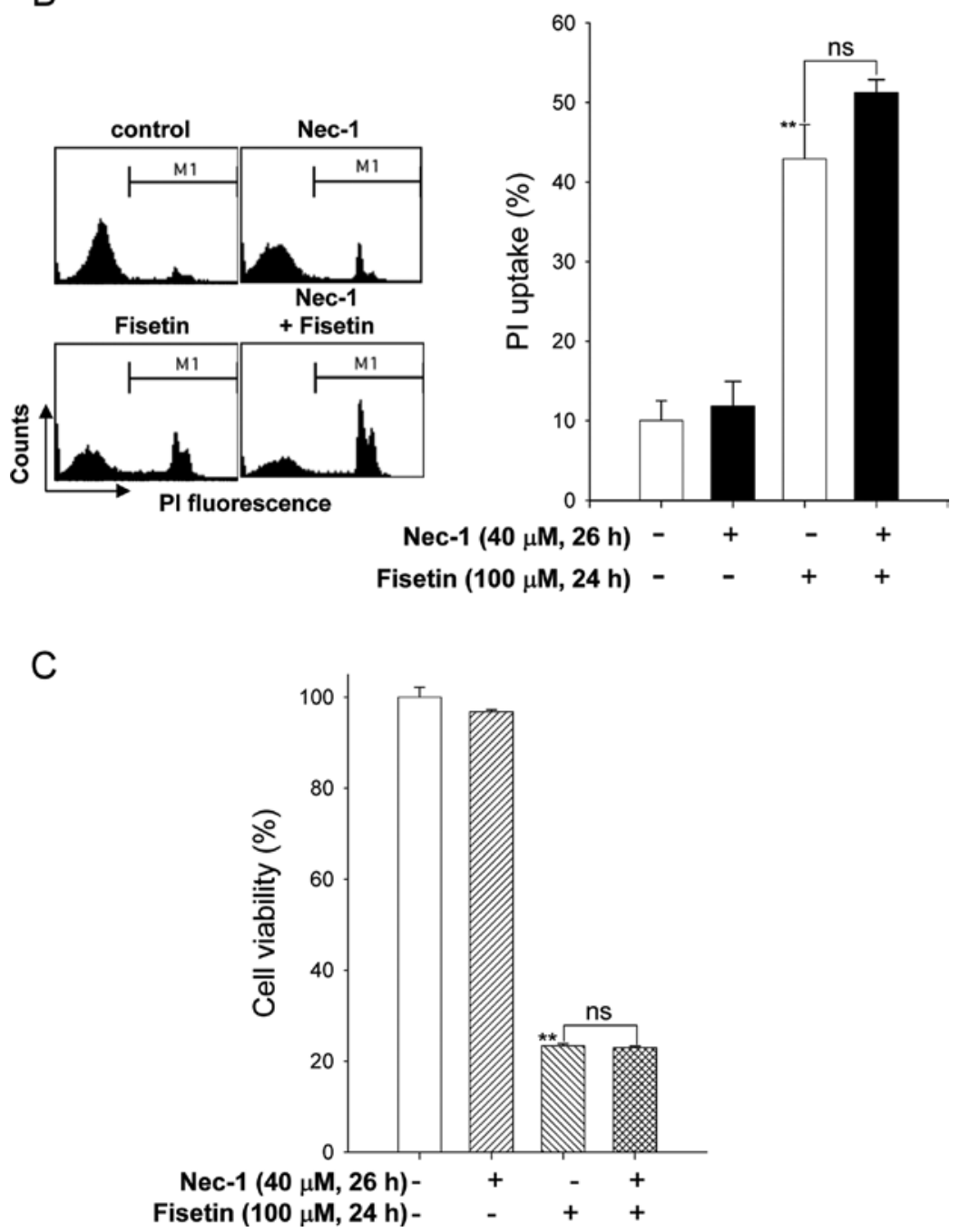

Figure 2. Fisetin does not induce necroptosis in MCF-7 cells. (A) MCF-7 cells were treated with $100 \mu \mathrm{M}$ fisetin for the indicated time periods or with $200 \mu \mathrm{M}$ $\mathrm{H}_{2} \mathrm{O}_{2}$ for $1 \mathrm{~h}$, and the intracellular ATP levels were analyzed. (B) MCF-7 cells were pretreated with $40 \mu \mathrm{M} \mathrm{Nec}-1$ for $2 \mathrm{~h}$ and then exposed to $100 \mu \mathrm{M}$ fisetin for $24 \mathrm{~h}$. Measurement of plasma membrane integrity was performed by PI uptake analysis. (C) MCF-7 cells were pretreated with $40 \mu \mathrm{M} \mathrm{Nec}-1$ for $2 \mathrm{~h}$ and then exposed to $100 \mu \mathrm{M}$ fisetin for $24 \mathrm{~h}$. The cytotoxicity was examined by MTT assay. The representative ${ }^{* *} \mathrm{p}<0.01$ indicates significant differences between fisetin-treated and untreated samples.

was observed in fisetin-treated MCF-7 cells (Fig. 3C and D), and pretreatment of $\mathrm{z}$-VAD-fmk further abrogated the cleavage of caspase-7 and PARP (Fig. 3E).
Fisetin induces mitochondrial depolarization and p53-independent cell death in MCF-7 cells. Apoptosis is induced via two main routes involving either mitochondria-mediated caspase-9 
A
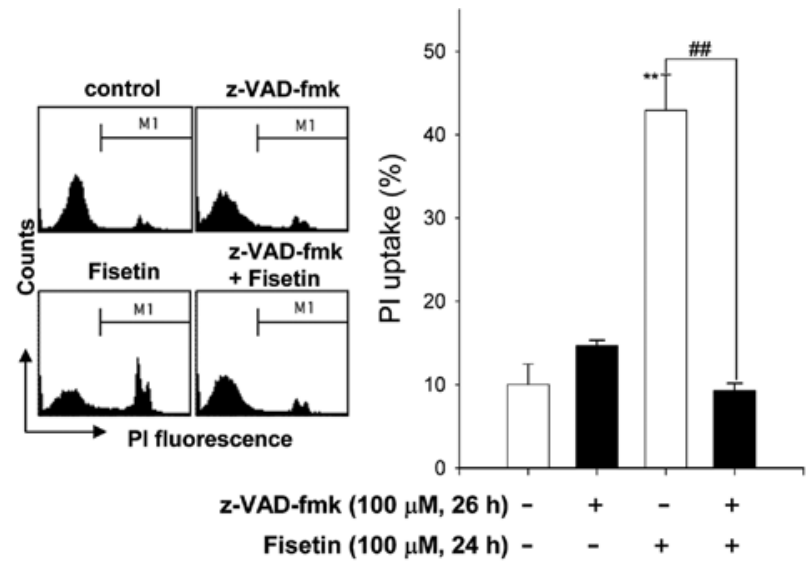

B

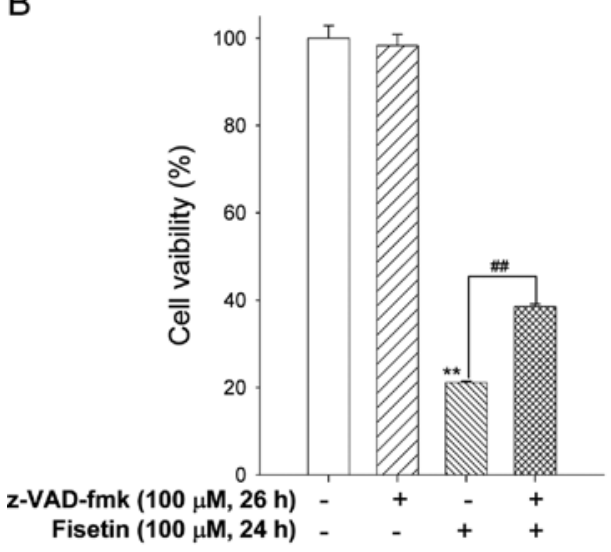

D

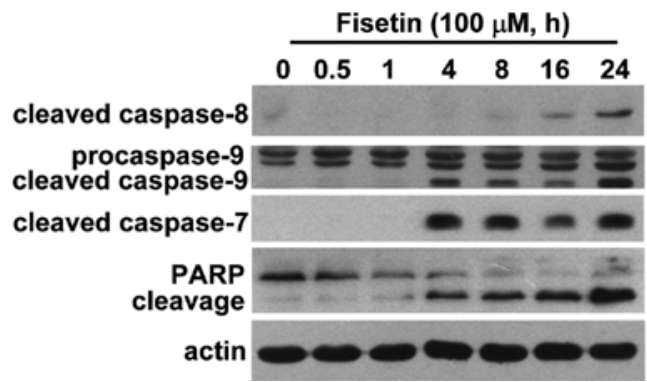

C

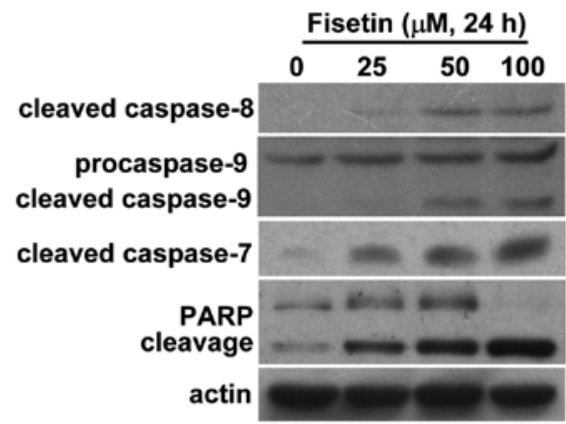

E

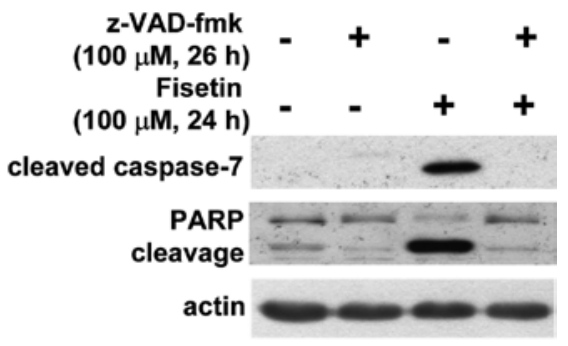

Figure 3. Fisetin induced caspase-dependent cell death in MCF-7 cells. MCF-7 cells were pretreated with $100 \mu \mathrm{M}$ z-VAD-fmk for $2 \mathrm{~h}$ then exposed to $100 \mu \mathrm{M}$ fisetin for $24 \mathrm{~h}$. The plasma membrane integrity was examined by PI uptake (A). The cytotoxicity was examined by MTT assay (B). The representative *" $<0.01$ indicates significant differences between fisetin-treated and untreated samples; ${ }^{\# \#} \mathrm{p}<0.01$ indicates a significant difference between the samples treated with fisetin alone and those pretreated with z-VAD-fmk. In (C), MCF-7 cells were treated with 25, 50 and $100 \mu \mathrm{M}$ fisetin. In (D), MCF-7 cells were treated with $100 \mu \mathrm{M}$ fisetin for 0-24 h. In (E), MCF-7 cells were pretreated with $100 \mu \mathrm{M} \mathrm{z}$-VAD-fmk for $2 \mathrm{~h}$ then exposed to $100 \mu \mathrm{M}$ fisetin for $24 \mathrm{~h}$. The cell lysates were subjected to immunoblotting using antibodies specific for caspase-7, $-8,-9$, PARP and actin.

activation (the intrinsic pathway) or the death receptors-induced activation of caspase-8 (the extrinsic pathway) (4). Our results indicated that activation of caspase- 9 occurs prior to that of caspase- 8 , which suggests that the mitochondrial pathway might be essential for fisetin-induced apoptosis (Fig. 3C and D). To this end, we investigated the degrees of mitochondrial depolarization with the measurements of the decreased rates of MMP in MCF-7 cells, which was determined by the fluorescence of the cationic lipophilic dye JC-1. On the other hand, cells treated with carbonyl cyanide 3-chlorophenylhydrazone (CCCP), an MMP disrupter, was used as a positive control. As shown in Fig. 4A, fisetin induced significant disruption of MMP in a concentrationdependent manner in MCF-7 cells. Reversely, the mitochondrial depolarization was efficiently inhibited by z-VAD-fmk (Fig. 4B).

p53 is a tumor suppressor protein that participates in the intrinsic apoptosis pathway by interacting with Bcl-2 family proteins to induce mitochondrial outer membrane permeabilization (27). The elevated expressing levels of phospho-Ser15 and total p53 protein induced by fisetin (Fig. 4C) further prompted us to investigate the involvement of p53 in fisetin-induced apop- 
A
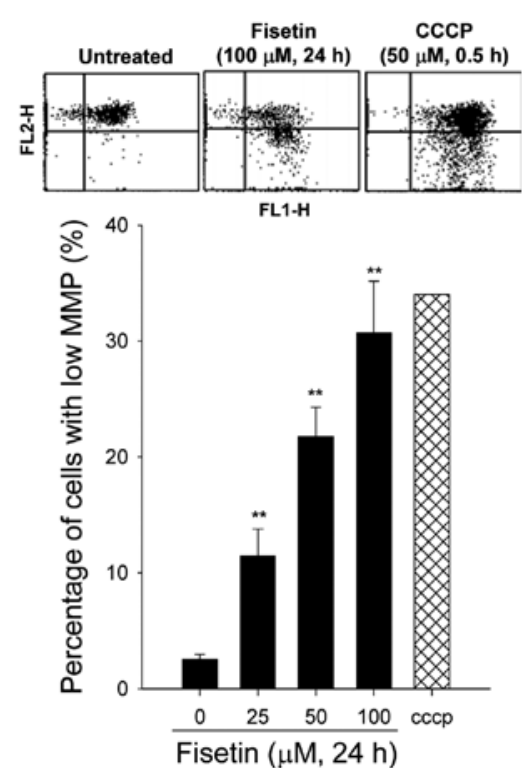

C

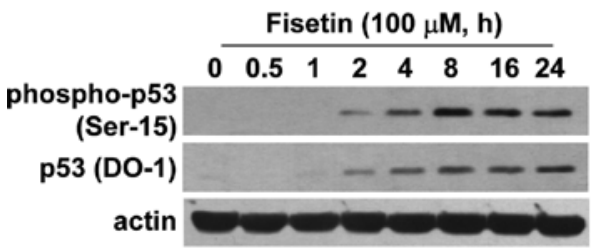

B

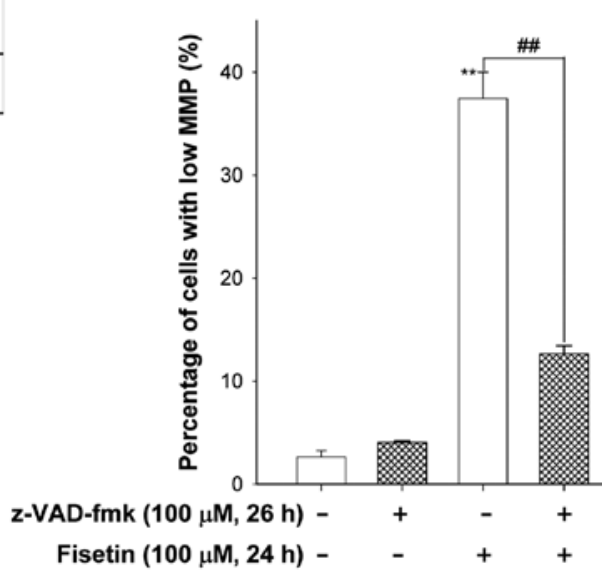

D

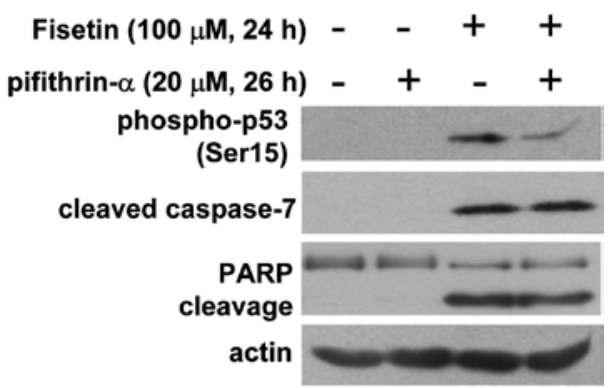

E

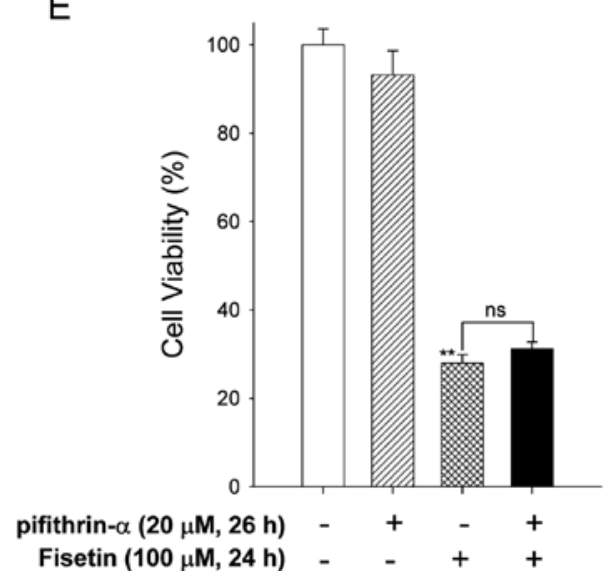

Figure 4. Fisetin induced mitochondrial depolarization and p53-independent cell death in MCF-7 cells. In (A), MCF-7 cells were treated with $100 \mu \mathrm{M}$ fisetin for $24 \mathrm{~h}$ or $50 \mu \mathrm{M} \mathrm{CCCP}$ for $1 \mathrm{~h}$. In (B), MCF-7 cells were pretreated with $100 \mu \mathrm{M} \mathrm{z}$-VAD-fmk for $2 \mathrm{~h}$ and then exposed to $100 \mu \mathrm{M}$ fisetin for $24 \mathrm{~h}$. The mitochondrial membrane potential was examined by JC-1 staining. (C) MCF-7 cells were exposed to $100 \mu \mathrm{M}$ fisetin for 0 to $24 \mathrm{~h}$. The protein levels of phospho-p53 (Ser15) and p53 (DO-1) were analyzed by Western blotting. In, (D) and (E), MCF-7 cells were pretreated with $20 \mu \mathrm{M}$ pifithrin- $\alpha$ for $2 \mathrm{~h}$ before exposure to $100 \mu \mathrm{M}$ fisetin for $24 \mathrm{~h}$. The protein levels of phospho-p53 (Ser15), caspase-7, PARP and actin were analyzed by Western blotting (D). The cytotoxicity was examined by MTT assay (E). The representative $*$ p $<0.01$ indicates significant differences between fisetin-treated and untreated samples.

tosis in MCF-7 cells. To verify such a possibility, the effects of p53 inhibitor pifithrin- $\alpha$ on fisetin-induced caspase-7 activation and PARP cleavage were monitored. As expected, pretreatment of pifithrin- $\alpha$ effectively blocked fisetin-induced p53 phosphorylation in MCF-7 cells. However, the result indicated fisetin-induced cleavage of caspase-7 and PARP was unaltered (Fig. 4D). In addition, inhibition of p53 activation did not reduce fisetin-induced cytotoxicity in MCF-7 cells (Fig. 4E). These result strongly supported that p53 did not participate in fisetininduced apoptosis.

Fisetin inhibits autophagy in MCF-7 cells. The removal or functional inhibition of apoptotic essential proteins can activate autophagy or cause a switch from the apoptotic default pathway 
A

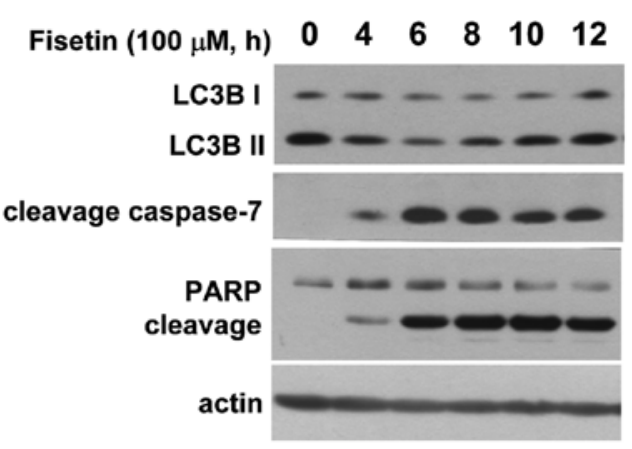

B

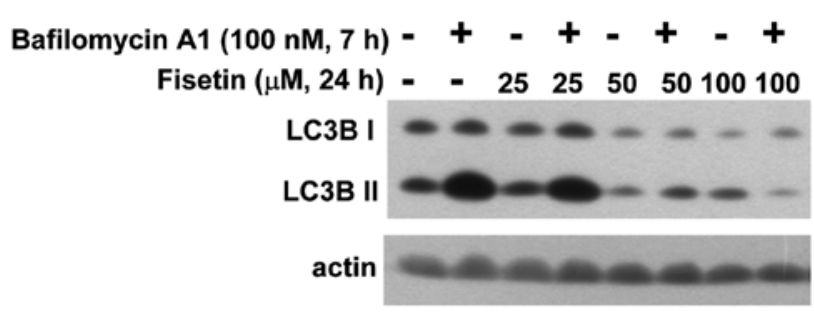

C

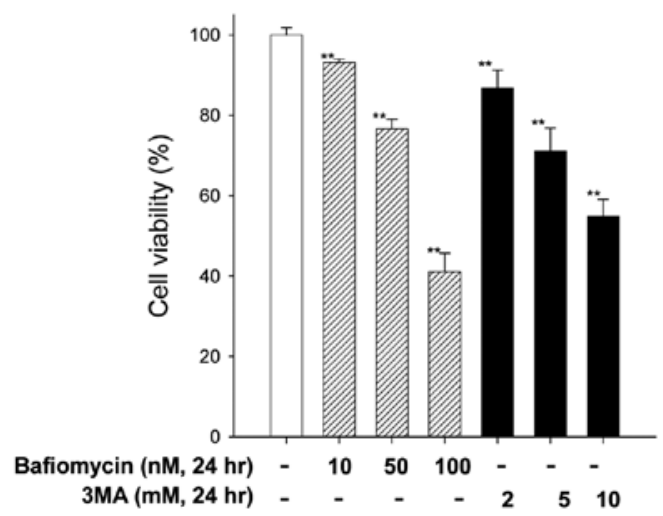

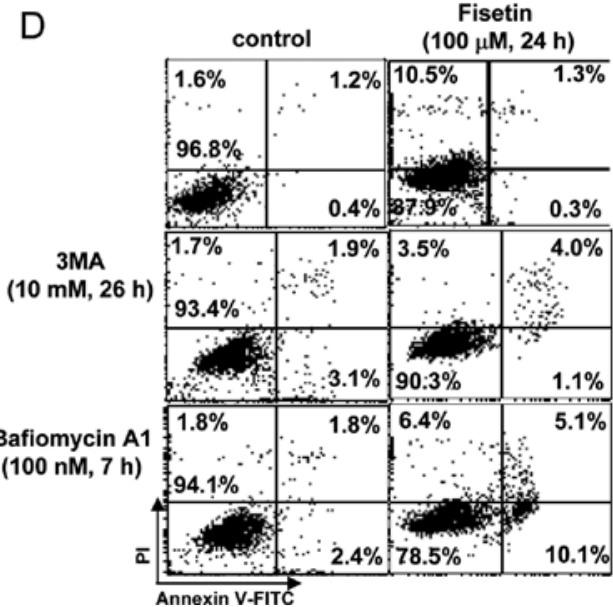

Figure 5. Fisetin inhibited autophagy in MCF-7 cells. In (A), MCF-7 cells were treated with $100 \mu \mathrm{M}$ fisetin for 0 to $12 \mathrm{~h}$. In (B), MCF-7 cells were treated with 25,50 or $100 \mu \mathrm{M}$ fisetin for $24 \mathrm{~h}$ and then exposed to $100 \mathrm{nM}$ bafilomycin A1 for the last $7 \mathrm{~h}$. The lysates were subjected to immunoblotting using antibodies specific for LC3B, caspase-7, PARP, and actin. (C) MCF-7 cells were treated with 10, 50, $100 \mathrm{nM}$ bafilomycin A1 or 2, 5, $10 \mathrm{mM} 3-\mathrm{MA}$ for $24 \mathrm{~h}$. The cell viability was examined by MTT assay. The representative ${ }^{* *} \mathrm{p}<0.01$ indicates significant differences between fisetin-treated and untreated samples. (D) MCF-7 cells were treated with $100 \mu \mathrm{M}$ fisetin for $24 \mathrm{~h}$ with or without $100 \mathrm{nM}$ bafilomycin A1 or $10 \mathrm{mM}$ 3-MA. Annexin V-FITC/PI double staining was performed.

to massive autophagy in response to cellular stress (28). To investigate whether fisetin induced autophagy in MCF-7 cells, the conversion of LC3-I to LC3-II in fisetin-treated MCF-7 cells was monitored by immunoblot analysis. LC3 exists in two forms, cytosolic LC3-I (autophagy-inactive) and processed LC3-II (autophagy-active), the latter of which is localized in autophagosome membranes, and whose levels are directly proportional to the levels of autophagic vacuoles $(29,30)$. Intriguingly, treatment of fisetin resulted in transient decrease of LC3-II expression (Fig. 5A) from 2 to $10 \mathrm{~h}$, which is accompanied with the cleavages of caspase- 7 and PARP, indicating fistein has negative effects on formation of autophagosomes. To verify this hypothesis, similar experiments were conduced using MCF7 cells with or without treatment of bafilomycin $\mathrm{A} 1$, a vacuolar-type $\mathrm{H}^{+}$-ATPase inhibitor that has been frequently used to block autophagosome-lysosome fusion. Consistently, LC3-II accumulation was efficiently impaired by fisetin in both the absence and presence of bafilomycin A1 (Fig. 5B), suggesting that endogenous formation of autophagy prompts cell survival in MCF-7 cells. To further address this possibility, MTT assay was employed to monitor cell viability of MCF-7 cells followed by the treatment of the autophagy inhibitors, 3-MA and bafilomycin A1. 3-MA, a class III PI3K inhibitor, was found to block autophagosome formation at the early stage of autophagic process. Both 3-MA and bafilomycin A1 significantly impaired the cell viability of MCF-7 cells. Moreover, 3-MA and bafilomycin A1 also increased the Annexin V/PI-positive population (Fig. 5D). Interestingly, 


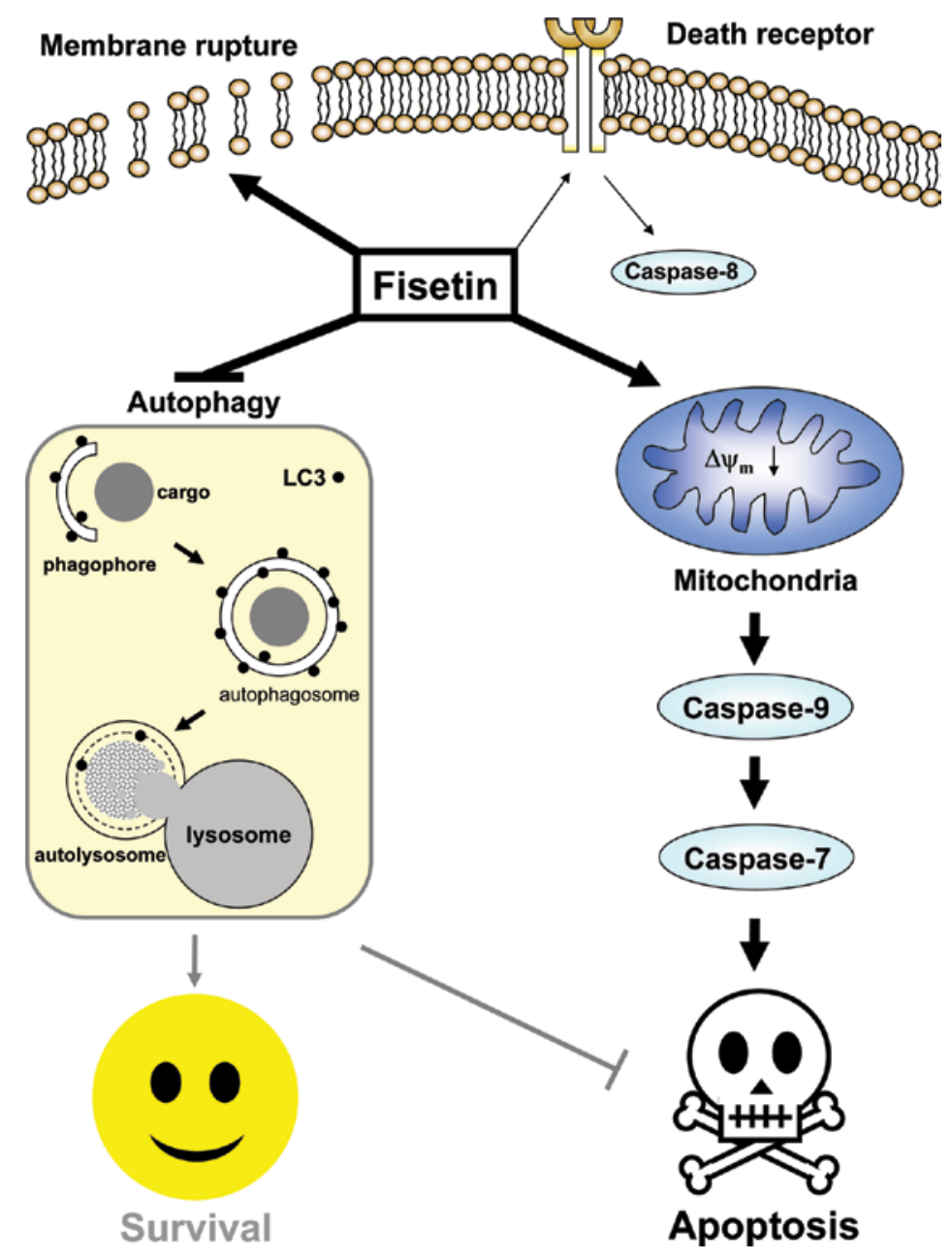

Figure 6. Proposed model of the anticancer effects induced by fisetin in MCF-7 cells.

combination of 3-MA or bafilomycin A1 with fisetin augmented the Annexin V/PI-positive population (Fig. 5D). These results indicated that inhibition of autophagy readily contributes to the anticancer effect of fisetin.

\section{Discussion}

Resistance of cancer cells to chemotherapy is a major clinical obstacle to the success of cancer therapy (31). A number of mechanisms by which human breast cancer cells become resistant to chemotherapeutic agents have been described (32). For example, lack of caspase-3 in MCF-7 cells leads to loss of the ability to carry out normal apoptosis and ultimately offers a possible mechanism to elicit chemoresistance $(19,20)$. Moreover, MDA-MB-231 cells that possess normal caspase-3 activity have been found to be more sensitive to chemotherapy drugs than MCF-7 cells (33-35). In contrast, here we demonstrated striking evidence showing MCF-7 cells were more susceptible to fisetininduced cytoxicity than MDA-MB-231 cells, indicating that fisetin treatment could suppress the chemoresistance produced by MCF-7 cells. Fisetin induced caspase-dependent cell death was characterized by the appearance of several apoptotic features, including the loss of plasma membrane integrity, mitochondrial depolarization, caspase-7, -8 and -9 activation, and PARP cleavage with the exception that DNA fragmentation and PS externalization were not observed. These results indicate that fisetin-induced cell death is distinct from the typical type of apoptosis.

Although caspase-3 has been shown to play a pivotal role in apoptotic events, such as DNA fragmentation and membrane blebbing, deficiency of caspase-3 does not affect Bax-induced levels of PARP cleavage, caspase- 6 activation, or lamin B cleavage (36). MCF-7 cells that lack caspase-3 undergo caspasedependent apoptotic cell death, with caspase-7 activation, PARP cleavage and DNA fragmentation being detected following $\mathrm{Pt}\left(\mathrm{O}, \mathrm{O}^{\prime}\right.$-acac) $(\gamma$-acac)(DMS) treatment (37), and the absence of DNA fragmentation and $\alpha$-fodrin cleavage following treatment with etoposide or doxorubicin (38), suggesting that the functional redundancy is evolutionarily maintained within the members of the caspase family (39). Although caspase- 3 and -7 have been shown to share some common substrates, they exhibit differential activities toward multiple substrate proteins (40). In particular, caspase- 3 is found to be more critical for induction of certain apoptotic events such as DNA fragmentation $(20,41)$. Our results further revealed that fisetin induces a novel type of apoptosis specified with both caspase-7 activation and mito- 
chondrial depolarization but those typical apoptotic features such as DNA fragmentation or PS externalization were not observed.

In addition to the basic role in the turnover of proteins and organelles, autophagy is observed under several pathological conditions, including myopathy, neuronal degeneration, infectious disease, and cancer $(42,43)$. Both blockage and induction of autophagy have been reported to be linked to tumor growth. The impacts of autophagy appears to vary with the intrinsic environmental properties of the tumor cells (44). Increasing evidence indicates that autophagy facilitates the resistance of cancer cells against chemotherapy and radiation (45). Recently, fisetin has been found to induce autophagic cell death through suppression of the mTOR signaling pathway in prostate cancer cells (46). However, several flavonoids, including fisetin, have been shown to have autophagy-inhibitory effects (47). In this study, we also demonstrated fisetin is a potent inhibitor of autophagy. Moreover, the autophagy inhibitors 3-MA and bafilomycin A1 was shown to induce cell death in MCF-7 cells. We propose that autophagy might play a protective role in fisetin-induced cell death in MCF-7 cells.

Caspase-independent cell death pathways are important safeguard mechanisms to protect organisms against unwanted and potential harmful cells when classical apoptotic routes triggered by caspases are blocked (48). Although early events involved in the apoptotic pathway are functioning actively in MCF-7 cells, caspase-3 deficiency might ultimately contribute to chemoresistance (49). Anticancer agents that elicit alternative forms of cell death turn out as promising candidates to be applied in the future development of anticancer therapy. Mutation of the p53 gene is frequently observed in human breast cancers and has been considered as another major cause involved in chemotherapy and radiotherapy resistance (50). Nonetheless, our results indicate that fisetin induced cell death is independent of p53. Consequently, the functional feature of fisetin to induced cell death in cancer cells is characterized by its ability to overcome the drug resistance elicited by defects of caspase-3 and/or p53-mediated cell death pathways.

Taken together, our results demonstrated that the natural flavonoid fisetin exhibits a selective anticancer activity in caspase-3-deficient MCF-7 cells through induction of an atypical form of apoptosis and inhibition of autophagy. Fisetin-induced apoptosis is associated with plasma membrane ruptures, mitochondrial depolarization, and activation of caspase-8, -9 , and -7 (Fig. 6). These results provide supporting evidence to allow fisetin being recognized as a potential chemotherapeutic agent for human breast cancer.

\section{Acknowledgements}

This study was supported by a grant from the National Science Council, Taiwan (NSC 99-2314-B-320-004-MY3). We acknowledge Dr Emma Carter for the copyediting of the manuscript.

\section{References}

1. Kroemer G, Galluzzi L, Vandenabeele P, et al: Classification of cell death: recommendations of the Nomenclature Committee on Cell Death 2009. Cell Death Differ 16: 3-11, 2009.

2. Majno G and Joris I: Apoptosis, oncosis, and necrosis. An overview of cell death. Am J Pathol 146: 3-15, 1995.
3. Hengartner MO: The biochemistry of apoptosis. Nature 407: 770-776, 2000.

4. Hotchkiss RS, Strasser A, McDunn JE and Swanson PE: Cell death. N Engl J Med 361: 1570-1583, 2009.

5. Meijer AJ and Dubbelhuis PF: Amino acid signalling and the integration of metabolism. Biochem Biophys Res Commun 313: 397-403, 2004

6. Levine B: Cell biology: autophagy and cancer. Nature 446: 745-747, 2007.

7. Parkin DM, Pisani P and Ferlay J: Estimates of the worldwide incidence of eighteen major cancers in 1985. Int J Cancer 54: 594-606, 1993

8. Russo J, Yang X, Hu YF, et al: Biological and molecular basis of human breast cancer. Front Biosci 3: D944-D960, 1998.

9. Andres AC and Strange R: Apoptosis in the estrous and menstrual cycles. J Mammary Gland Biol Neoplasia 4: 221-228, 1999.

10. Simstein R, Burow M, Parker A, Weldon C and Beckman B: Apoptosis, chemoresistance, and breast cancer: insights from the MCF-7 cell model system. Exp Biol Med (Maywood) 228: 995-1003, 2003.

11. Fisher DE: Apoptosis in cancer therapy: crossing the threshold. Cell 78: 539-542, 1994.

12. Higa S, Hirano T, Kotani M, et al: Fisetin, a flavonol, inhibits TH2-type cytokine production by activated human basophils. J Allergy Clin Immunol 111: 1299-1306, 2003.

13. Khan N, Afaq F, Syed DN and Mukhtar H: Fisetin, a novel dietary flavonoid, causes apoptosis and cell cycle arrest in human prostate cancer LNCaP cells. Carcinogenesis 29: 1049-1056, 2008.

14. Chen YC, Shen SC, Lee WR, et al: Wogonin and fisetin induction of apoptosis through activation of caspase 3 cascade and alternative expression of p21 protein in hepatocellular carcinoma cells SK-HEP-1. Arch Toxicol 76: 351-359, 2002.

15. Lu X, Jung J, Cho HJ, et al: Fisetin inhibits the activities of cyclindependent kinases leading to cell cycle arrest in HT-29 human colon cancer cells. J Nutr 135: 2884-2890, 2005.

16. Suh Y, Afaq F, Johnson JJ and Mukhtar H: A plant flavonoid fisetin induces apoptosis in colon cancer cells by inhibition of COX2 and Wnt/EGFR/NF-kappaB-signaling pathways. Carcinogenesis 30: 300-307, 2009.

17. Haddad AQ, Venkateswaran V, Viswanathan L, Teahan SJ, Fleshner NE and Klotz LH: Novel antiproliferative flavonoids induce cell cycle arrest in human prostate cancer cell lines. Prostate Cancer Prostatic Dis 9: 68-76, 2006.

18. Chen WS, Yu YC,Lee YJ, Chen JH,Hsu HY and Chiu SJ: Depletion of securin induces senescence after irradiation and enhances radiosensitivity in human cancer cells regardless of functional p53 expression. Int J Radiat Oncol Biol Phys 77: 566-574, 2010.

19. Devarajan E, Sahin AA, Chen JS, et al: Down-regulation of caspase 3 in breast cancer: a possible mechanism for chemoresistance. Oncogene 21: 8843-8851, 2002

20. Janicke RU, Sprengart ML, Wati MR and Porter AG: Caspase-3 is required for DNA fragmentation and morphological changes associated with apoptosis. J Biol Chem 273: 9357-9360, 1998.

21. Degterev A, Huang Z, Boyce M, et al: Chemical inhibitor of nonapoptotic cell death with therapeutic potential for ischemic brain injury. Nat Chem Biol 1: 112-119, 2005.

22. Eguchi Y, Shimizu S and Tsujimoto Y: Intracellular ATP levels determine cell death fate by apoptosis or necrosis. Cancer Res 57: 1835-1840, 1997.

23. Cohen GM: Caspases: the executioners of apoptosis. Biochem J 326 (Pt 1): 1-16, 1997.

24. Fernandes-Alnemri T, Takahashi A, Armstrong R, et al: Mch3, a novel human apoptotic cysteine protease highly related to CPP32. Cancer Res 55: 6045-6052, 1995.

25. Thornberry NA, Rano TA, Peterson EP, et al: A combinatorial approach defines specificities of members of the caspase family and granzyme B. Functional relationships established for key mediators of apoptosis. J Biol Chem 272: 17907-17911, 1997.

26. Duan H, Chinnaiyan AM, Hudson PL, Wing JP, He WW and Dixit VM: ICE-LAP3, a novel mammalian homologue of the Caenorhabditis elegans cell death protein Ced-3 is activated during Fas- and tumor necrosis factor-induced apoptosis. J Biol Chem 271: $1621-1625,1996$.

27. Vaseva AV and Moll UM: The mitochondrial p53 pathway. Biochim Biophys Acta 1787: 414-420, 2009.

28. Maiuri MC, Zalckvar E, Kimchi A and Kroemer G: Self-eating and self-killing: crosstalk between autophagy and apoptosis. Nat Rev Mol Cell Biol 8: 741-752, 2007.

29. Kabeya Y, Mizushima N, Ueno T, et al: LC3, a mammalian homologue of yeast Apg8p, is localized in autophagosome membranes after processing. EMBO J 19: 5720-5728, 2000. 
30. Mizushima N: Methods for monitoring autophagy. Int J Biochem Cell Biol 36: 2491-2502, 2004.

31. Lehnert M: Chemotherapy resistance in breast cancer. Anticancer Res 18: 2225-2226, 1998.

32. Michor F, Nowak MA and Iwasa Y: Evolution of resistance to cancer therapy. Curr Pharm Des 12: 261-271, 2006.

33. MaZ, Molavi O, Haddadi A,Lai R, Gossage RA and Lavasanifar A: Resveratrol analog trans 3,4,5,4'-tetramethoxystilbene (DMU-212) mediates anti-tumor effects via mechanism different from that of resveratrol. Cancer Chemother Pharmacol 63: 27-35, 2008.

34. Wang L, Li Z, Wang C, et al: E-cadherin decreased human breast cancer cells sensitivity to staurosporine by up-regulating Bcl-2 expression. Arch Biochem Biophys 481: 116-122, 2009.

35. Xie SQ, Zhang ZQ, Hu GQ, Xu M and Ji BS: HL-37, a novel anthracene derivative, induces $\mathrm{Ca}(2+)$-mediated apoptosis in human breast cancer cells. Toxicology 254: 68-74, 2008.

36. Kagawa S, Gu J, Honda T, et al: Deficiency of caspase-3 in MCF7 cells blocks Bax-mediated nuclear fragmentation but not cell death. Clin Cancer Res 7: 1474-1480, 2001.

37. Muscella A, Calabriso N, Fanizzi FP, et al: $\left[\mathrm{Pt}\left(\mathrm{O}, \mathrm{O}^{\prime}-\mathrm{acac}\right)\right.$ (gammaacac)(DMS)], a new Pt compound exerting fast cytotoxicity in MCF-7 breast cancer cells via the mitochondrial apoptotic pathway. Br J Pharmacol 153: 34-49, 2008.

38. Janicke RU, Engels IH, Dunkern T, Kaina B, Schulze-Osthoff K and Porter AG: Ionizing radiation but not anticancer drugs causes cell cycle arrest and failure to activate the mitochondrial death pathway in MCF-7 breast carcinoma cells. Oncogene 20: 5043-5053, 2001.

39. Janicke RU, Ng P, Sprengart ML and Porter AG: Caspase-3 is required for alpha-fodrin cleavage but dispensable for cleavage of other death substrates in apoptosis. J Biol Chem 273: 15540-15545, 1998.
40. Walsh JG, Cullen SP, Sheridan C, Luthi AU, Gerner C and Martin SJ: Executioner caspase-3 and caspase-7 are functionally distinct proteases. Proc Natl Acad Sci USA 105: 12815-12819, 2008.

41. Cao G, Pei W, Lan J, et al: Caspase-activated DNase/DNA fragmentation factor 40 mediates apoptotic DNA fragmentation in transient cerebral ischemia and in neuronal cultures. J Neurosci 21: 4678-4690, 2001.

42. Larsen KE and Sulzer D: Autophagy in neurons: a review. Histol Histopathol 17: 897-908, 2002.

43. Nishino I: Autophagic vacuolar myopathies. Curr Neurol Neurosci Rep 3: 64-69, 2003.

44. Mathew R, Karantza-Wadsworth V and White E: Role of autophagy in cancer. Nat Rev Cancer 7: 961-967, 2007.

45. Chen S, Rehman SK, Zhang W, Wen A, Yao L and Zhang J: Autophagy is a therapeutic target in anticancer drug resistance. Biochim Biophys Acta 1806: 220-229, 2010.

46. Suh Y, Afaq F, Khan N, Johnson JJ, Khusro FH and Mukhtar H: Fisetin induces autophagic cell death through suppression of mTOR signaling pathway in prostate cancer cells. Carcinogenesis 31: 1424-1433, 2010.

47. Gordon PB, Holen I and Seglen PO: Protection by naringin and some other flavonoids of hepatocytic autophagy and endocytosis against inhibition by okadaic acid. J Biol Chem 270: 5830-5838, 1995.

48. Broker LE, Kruyt FA and Giaccone G: Cell death independent of caspases: a review. Clin Cancer Res 11: 3155-3162, 2005.

49. Yang XH, Sladek TL, Liu X, Butler BR, Froelich CJ and Thor AD: Reconstitution of caspase 3 sensitizes MCF-7 breast cancer cells to doxorubicin- and etoposide-induced apoptosis. Cancer Res 61: 348-354, 2001.

50. Scata KA and El-Deiry WS: p53, BRCA1 and breast Cancer chemoresistance. Adv Exp Med Biol 608: 70-86, 2007. 